Meta

Journal des traducteurs

Translators' Journal

\title{
Table ronde : l'adaptation publicitaire, oui ou non?
}

\section{Marcel Paré, Roger Boivineau, Jean-François Pelletier, Gérard Normandin et Lucien Roy}

Volume 17, numéro 1, mars 1972

L’adaptation publicitaire

URI : https://id.erudit.org/iderudit/003785ar

DOI : https://doi.org/10.7202/003785ar

Aller au sommaire du numéro

Éditeur(s)

Les Presses de l'Université de Montréal

ISSN

0026-0452 (imprimé)

1492-1421 (numérique)

Découvrir la revue

Citer cet article

Paré, M., Boivineau, R., Pelletier, J.-F., Normandin, G. \& Roy, L. (1972). Table

ronde : l'adaptation publicitaire, oui ou non? Meta, 17(1), 29-46.

https://doi.org/10.7202/003785ar d'utilisation que vous pouvez consulter en ligne. 


\section{Table ronde: l'adaptation publicitaire, oui ou non?}

1. M. Paré. - Tout le monde est d'accord, je crois, pour considérer l'adaptation publicitaire comme une solution de remplacement de la création, particulièrement dans le Québec. Cette adaptation est parfois réalisable; d'autres fois elle est impossible. Dans certains cas, elle est acceptable ; dans d'autres elle est à rejeter. Que faut-il pour qu'un texte soit adaptable et que son adaptation soit acceptable?

2. R. Boivineau. - Quand on parle d'adaptabilité et d'inadaptabilité, car je pense que la question revient à cela, la première distinction qui s'impose porte sur la nature des textes. Distinguons donc les annonces des autres textes publicitaires. Tout ce qui n'est pas purement annonce est relativement facile à adapter. Je pense en particulier aux catalogues, et même aux annonces de détaillants, qui sont en somme des annonces-catalogues, ou encore aux circulaires de publicité directe et à la plupart des prospectus et dépliants.

Quant aux annonces, certaines d'entre elles sont beaucoup plus faciles à adapter que d'autres. En publicité industrielle en particulier on obtient très facilement une adaptation acceptable. Vendre un chariot élévateur, cela revient à peu près à la même chose dans toutes les langues. Évidemment, l'annonce originale peut comporter un accrochage plus ou moins savant, contenant un jeu de mots, mais le sujet ne prête pas beaucoup à la fantaisie et on a à peu près toujours les mêmes arguments à faire valoir, et de la même façon, dans la langue de départ et dans la langue d'arrivée. Les annonces de prestige, la publicité de notoriété, sont également assez facilement adaptables. Mais pour les produits de grande consommation, l'adaptation est beaucoup plus difficile et, dans certains cas, on a affaire à des textes tout simplement inadaptables ou dont l'adaptation est à déconseiller si on ne veut pas que le texte sente la traduction, ce qui est évidemment à éviter à tout prix. C'est le cas, entre autres, des annonces qui imposent pour le titre un graphisme impossible à respecter, qui lient un jeu de mots à une illustration, qui comportent des éléments socioculturels incompatibles avec le public visé. 
3. J.-F. Pelletier. - En effet, le facteur socioculturel influe fortement, non seulement sur l'alternative adaptation/traduction, mais sur tout le secteur de la rédaction publicitaire - du moins, telle qu'elle se pratique au Québec. Car, justement, il faut voir comment les choses se passent. Parler de l'adaptation, « en général », est intéressant mais, dans la pratique, rime à peu de chose. Parlons plutôt de l'adaptation et de la traduction telles qu'elles se font ici : soit, à peu près toujours de l'anglais au français - ou, plus exactement, de l'américain au français. Et «traduire », dans son sens fort, ce n'est pas traduire que le mot mais c'est encore davantage traduire l'idée. Partant de là, il faut reconnaître que le rédacteur du texte d'origine, parce qu'anglo-américain, a utilisé, dans son annonce, non seulement la langue anglo-américaine mais aussi une conceptualisation angloaméricaine basée sur un système de métaphores anglo-américain, une échelle de valeurs anglo-américaine, une mythologie anglo-américaine. Mais notre rédacteur à nous, étant franco-québécois, devra transposer le tout en des équivalences franco-québécoises : et la langue, et la pensée, et les valeurs, et les métaphores, et la mythologie.

Je cherche donc à souligner, et avec force, que parler de «traduire un texte d'une langue à une autre » constitue, en fait, une fausseté aussi dangereuse que courante. Car une langue est bien autre chose qu'un simple système de communication ; c'est en quelque sorte une façon de penser, une façon de vivre, une façon de juger les hommes et les choses. Comme 1'Anglo-Américain (terme qui, pour moi, englobe l'Anglo-Canadien) est parti de sa langue, de ses valeurs et de sa mythologie pour établir l'annonce anglaise, le publicitaire franco-québécois devra établir une annonce française où nos consommateurs retrouvent notre langue, nos valeurs et notre mythologie. Bref le passage, de l'original anglo-américain à l'adaptation québécoise, ne se fera correctement que dans la mesure où il tiendra un compte rigoureux des divergences socioculturelles qui caractérisent chacune des deux sociétés en cause, l'américaine et la québécoise. Quand c'est assez facile c'est-à-dire quand le facteur socioculturel crée peu de «conflit » entre les deux textes - alors, une simple traduction peut souvent suffire. Mais quand les disparités socioculturelles s'avèrent considérables entre les deux textes - l'anglais qui existe et le français à naître - alors, on doit distancier davantage, et donc adapter plutôt que traduire. Du reste, cet écart socioculturel peut s'aggraver au point d'interdire toute transposition du texte en cause. Bref, la possibilité de traduire ou d'adapter est surtout déterminée par des considérations de cultures et de langues comparées; et parfois aussi, par des raisons purement techniques telles que l'impossibilité d'accorder un bon texte français à l'illustration conservée de l'original anglais.

Mais apprécier s'il est possible de traduire n'est pas tout ; restera à apprécier si on doit, ou non, traduire. Je m'explique. Outre la possibilité technique de traduire une annonce de l'américain au français, il y a aussi à juger de l'opportunité éthique de le faire. En termes plus simples, j'examine mon annonce anglaise et je me dis : les idées exprimées et les mots utilisés ne m'empêcheraient pas de traduire, mais serait-il moral de le faire ? Renversons la question : quand et comment pourrait-il être immoral de traduire? Il me paraît volontiers immoral de traduire une annonce si ce fait produit un ou plusieurs des effets suivants : trahir 
la langue d'arrivée (le français) en faussant son vocabulaire, sa syntaxe, son esprit ; trahir ou fausser le maniement de ses métaphores propres, de sa mythologie socioculturelle propre; trahir ou fausser le système particulier des valeurs de la société à laquelle cette annonce s'adresse.

Mais si j'illustrais par un exemple ou deux? Quand une annonce américaine prône le snobisme et le mimétisme social, représentés par l'idée que le consommateur must keep up with the Joneses, le publicitaire québécois a-t-il vraiment le droit moral de proposer ainsi cet «idéal » à ces compatriotes ? Car, il ne faut pas s'y tromper : dans le mobile du type to keep up with the Joneses, c'est le système de valeurs qui est invoqué - pour ne pas dire... attaqué. Problème délicat et important. Problème autrement fondamental que celui de résoudre la difficulté technique de transposer en anglais, par exemple, des expressions même difficiles comme «taper dans le mille» ou «tomber à pic». Car il faut voir clairement que la publicité en général, et la publicité anglo-américaine en particulier, s'avère le monde par excellence des mythes : mythe de la nouveauté, mythe de la mode, mythe de la jeunesse, mythe du confort, mythe du succès, mythe de la sexualité toute-puissante, mythe de l'enfant-roi, mythe de la propriété matérielle (quasi hélas! par opposition à la propriété intellectuelle ou morale...). Mythes et mobiles - arsenal de choix de la publicité contemporaine. Or, qu'on le veuille ou non, quand on accepte de traduire ou d'adapter une annonce, on accepte le plus souvent de transposer dans l'autre langue, non seulement des mots (ce qui finit par importer assez peu, en définitive !), mais de transposer aussi et surtout des idées, des valeurs et des mythes qui peuvent être carrément délétères pour notre société québécoise. Et quand ces mythes et valeurs sont projetés dans le public avec la force et le raffinement de la publicité moderne... !

$\mathrm{Au}$ Québec, peut-être plus que n'importe où ailleurs, on aurait grand tort de considérer la traduction publicitaire comme une fonction purement technique, où il suffit de surveiller la précision du mot, la justesse de l'image ou la rigueur de la syntaxe. C'est là où nous en étions, il y a vingt ou trente ans, l'ère des sottises carabinées du genre : This brief is machine washable que les sous-vêtements Watson traduisaient encore, il y a quelques mois, par : «Ce brevet est lavable à la machine. » Bon, mais, quand même, ère largement révolue. Aujourd'hui, le publicitaire franco-québécois a besoin, d'abord et avant tout, d'une grande et constante lucidité dans le maniement des réalités socioculturelles, plutôt que dans le maniement traditionnel des réalités langagières et conceptuelles.

Bref, aujourd'hui, la publicité québécoise devrait avoir dépassé, une fois pour toutes, le stade plutôt technique et transitoire de la traduction et de l'adaptation, pour accéder enfin au stade permanent et fondamental - celui des préoccupations socioculturelles, celui de la communication avec un milieu selon ses modes propres et en respectant intégralement ses valeurs essentielles. Autrement dit, de nos jours, les traducteurs publicitaires du Québec ont bien plus besoin de conscience sociale que de connaissances linguistiques et techniques... même si, jusqu'ici, ils n'ont épaté personne par un excès de telles connaissances.

4. R. Boivineau. - Excusez-moi de revenir sur la question de la «moralité » de la traduction, mais vous remettez absolument en question tous les fondements 
de la publicité et vous dépassez là le cadre de l'adaptation. Disons que l'adaptateur a pour rôle d'atténuer certains aspects excessifs de la publicité originale, dans le ton de l'annonce par exemple, dans les exagérations hyperboliques - de moins en moins fréquentes, il faut le dire, dans le nouveau style publicitaire. Il se doit d'éliminer tout ce qui pourra nuire à la crédibilité du texte adapté auprès du public. Mais l'adaptateur ne doit pas à mon avis modifier les arguments invoqués sous prétexte qu'il ne les trouve pas conformes à sa morale personnelle. Les seuls cas où il pourrait le faire seraient ceux où ces arguments seraient en contradiction avec l'éthique professionnelle, ce qui est rarissime, ou seraient nuisibles au rendement de l'annonce, ce qui est beaucoup plus fréquent.

5. G. Normandin. - Oui, le raisonnement ne s'applique que dans la mesure où une exagération notoire pourrait nuire à la vente du produit ou du service. Je pense que notre fonction n'est pas une fonction moralisatrice, même si je déplore certains excès autant que tous les autres publicitaires. C'est une fonction de vente. Et en offrant le produit d'une façon outrancière, en jouant exagérément sur les sentiments de snobisme, je pense qu'on peut effectivement nuire à son succès et à son débit au Québec. Je me dois toutefois d'abonder dans le sens de M. Pelletier lorsqu'il parle de la métaphore. Mais, si vous le permettez, j'aimerais revenir à la question qui nous a été posée et changer le point de vue, c'est-à-dire considérer la chose lorsque le travail est terminé. Quand une adaptation est-elle acceptable, quand est-elle à rejeter ? Supposons que nous ayons devant nous une série d'adaptations et qu'il faille déterminer lesquelles sont acceptables et lesquelles sont à rejeter. Me plaçant de ce point de vue-là, je vous propose le critère suivant : si l'adaptation se reconnaît comme telle, je la trouve inacceptable; si l'on n'y reconnaît pas une traduction ou un calque de l'anglais ou de quelque autre langue, je pense qu'elle passe.

6. M. Paré. - Autrement dit, vous reconnaissez dans l'adaptation un cheminement de pensée en accord avec votre culture et en accord avec celle des lecteurs à qui est destiné le message. Vous n'y décelez pas des cheminements qui ne vous sont pas familiers, qui ne seront pas familiers aux lecteurs...

7. L. Roy. - Quand on juge l'adaptation publicitaire, je pense qu'il faut faire la distinction entre deux sortes de publicité. Il y a la publicité qui porte sur l'information et celle qui fait appel aux sentiments. L'une peut s'adapter plus facilement que l'autre. Il y a aussi la publicité, très fréquente, qui combine les deux. Elle présente des faits mais utilise aussi des arguments abstraits. La publicité, qui parle, par exemple, du perfectionnement d'un produit, fait souvent valoir des avantages qui peuvent intéresser autant le francophone que l'anglophone et je ne pense pas que l'adaptation d'un message annonçant une hausse d'intérêt bancaire de $6 \%$ à $7 \%$ porte atteinte à la culture franco-québécoise. Ce genre d'annonce peut s'adapter. Même si notre échelle de valeurs n'est pas tout à fait la même que celle des Canadiens anglophones, le consommateur québécois ne se désintéresse tout de même pas entièrement du confort matériel. Il ne faut pas, en matière de publicité, prendre notre culture pour ce qu'elle devrait être ou pour ce qu'elle a pu être. Elle aussi, je pense, a évolué. Le perfectionnement d'une voiture intéresse une multitude de gens quelle que soit leur langue. Une 
grande difficulté d'adaptation se présente quand, par exemple, une métaphore anglaise est reliée à une illustration. Une idée peut toujours s'exprimer dans une autre langue. Mais quand elle est combinée à une image, l'adaptation est souvent impossible. Il faut alors laisser tomber le message ou l'illustration ou les deux. Voilà un cas où il vaut mieux ne pas adapter.

8. G. Normandin. - Oui, c'est un carcan, c'est évident. Mais pour revenir à ce que Monsieur Roy disait tout à l'heure, quant à l'adaptabilité de la publicité d'information, je pense qu'on peut aller plus loin encore et considérer ce dont on habille cette information. Car presque toute information fournie par la publicité a quand même un caractère émotif. Sinon, la publicité se doit de lui en donner un. Je ne parle pas de fiches techniques et de choses du genre. Mais considérez une augmentation du taux d'intérêt, par exemple. La publicité se doit d'associer cette hausse à un avantage quelconque. C'est ce que j'appelle l'habit de l'information, et pour l'adaptateur, pour le traducteur, cet habit est excessivement important. Sur le strict plan de l'information, je suis tout à fait d'accord mais sur le plan émotif qu'implique cette information, je crois qu'il faut étudier sérieusement la situation avant de déterminer si oui ou non on doit passer outre.

9. M. Paré. - Vous avez mentionné deux termes au cours de votre intervention, adaptateur et traducteur et je pense précisément que c'est à ce palier-là que se situe une importante démarcation. Moi, je confonds les deux, le traducteur et l'adaptateur, parce que le traducteur qui est compétent pour traduire une annonce de publicité ne peut pas ne pas être dans une certaine mesure un adaptateur même s'il n'a pas la même liberté que son collègue qui est dans une agence de publicité et qui a à sa disposition une abondance d'information que l'autre n'a pas. C'est plus délicat peut-être pour le traducteur de pousser trop loin son adaptation, mais est-ce que selon vous il ne doit pas d'abord essayer d'obtenir toute l'information possible et c'est à partir de ce moment-là seulement qu'il peut se permettre une certaine mesure de liberté.

10. G. Normandin. - On a donné depuis quelques années au terme «traducteur $»$ surtout dans le monde publicitaire, un sens péjoratif et c'est pour cela qu'on emploie de préférence adaptation à traduction. Mais ce que vous dites est très vrai et, pour moi d'ailleurs, le seul traducteur publicitaire qui mérite ce titre est effectivement un adaptateur.

11. L. Roy. - En publicitê, la traduction qui a été critiquée n'était pas, en fait, de la traduction. C'était de l'anglais transposé en mauvais français. Les traducteurs n'avaient pas la formation requise. C'est pour cette raison qu'on n'aime plus parler de traduction. Mais à y penser, une bonne traduction vaut mieux qu'une mauvaise adaptation.

12. J.-F. Pelletier. - D'accord avec vous, M. Roy. Comme le Québec traîne un lourd passé de fort vilaines traductions, il a fini par rejeter la traduction en tant que telle, au lieu de distinguer entre la bonne traduction - la seule vraie et la mauvaise, celle qui, au fond, n'en est pas du tout. Ce qui, à la longue, nous amena au phénomène connu - celui de garder la chose, mais d'en changer le nom. Ft c'est ainsi que «traduction» devint « adaptation »... Reste, aussi, qu'on 
voyait sans doute (ne serait-ce qu'obscurément) dans l'adaptation une espèce de moyen terme entre l'esclavage de la traduction et la liberté de la création. Car, au fond, ce qu'on cherchait (encore une fois, obscurément), c'était justement la création, débarrassée des servitudes de la traduction et de l'adaptation.

Ce qui me permet d'enchaîner en disant que si votre question se lit « L'adaptation, solution de remplacement de la création $»$, mois je vous dirai, M. Paré, qu'à mon avis l'adaptation ne remplace rien du tout : ce n'est qu'un mal nécessaire. Car si le Québec était en situation existentielle normale, personne ici ne songerait (du moins, de façon habituelle) à traduire ou à adapter des annonces, mais poserait l'acte évident et normal de créer nos publicités. Aussi me paraît-il d'une pénible évidence que traduction et adaptation, même quand elles sont éblouissantes d'habileté, ne sont que des opérations de rechange, des ersatz, des maux nécessaires, quoi! Et il ne faudra pas oublier, ici, de distinguer carrément entre la traduction publicitaire, née d'un «besoin» imaginaire et faux, et la traduction littéraire, procédant d'un besoin réel et vrai.

13. G. Normandin. - Je suis malheureusement en désaccord parce que je reconnais à la traduction publicitaire la même liberté d'être qu'à la traduction littéraire. Si on se place dans le contexte qui est celui de la vente pure, le but à atteindre étant mercantile, nous prenons des moyens appropriés. Il y a évidemment dans tout concepteur publicitaire un artiste ou un écrivain qui sommeille et qui voudrait s'exprimer, qui voudrait vivre en dehors de ce cadre mercantile. Mais nous y vivons et nous y gagnons notre vie. Vous avez parlé tout à l'heure de mal nécessaire. Je conçois que, pour un concepteur canadien-français, il soit beaucoup moins intéressant d'adapter les idées des autres que de créer à partir de ses propres idées. Toutefois, lorsqu'une équipe de création a travaillé aux ÉtatsUnis, à Toronto, en Europe pour produire des choses vraiment sensationnelles et éminemment adaptables, je pense que c'est du temps perdu que de mettre des concepteurs publicitaires devant le même problème et de leur demander d'arriver à une solution qui soit supérieure. La chose n'est pas impossible, mais si vous partiez du principe que la traduction est un mal nécessaire, vous diriez : « Ne traduisons pas les pièces de Shakespeare en français. Demandons à une équipe de dramaturges français de nous concevoir des pièces qui soient supérieures à celles de Shakespeare.»

14. J.-F. Pelletier. - Inutile de dire que je conteste, de la façon la plus catégorique, autant votre rejet des responsabilités éthiques du publicitaire, que votre prétention que la traduction littéraire cautionne le bien-fondé de la traduction publicitaire. Il me paraît évident que la traduction littéraire, elle, poursuit la fin bien légitime de communiquer à une société de langue « $\mathrm{A}$ 》 un fond culturel de langue « $\mathrm{B}$ »- langue qu'en général « $\mathrm{A}$ » entend peu ou pas. Ce qui n'a rien à voir avec une tentative ridicule de créer un Shakespeare français, qui, dans son aspect français, serait supérieur au Shakespeare anglais. Mais que, par contre, Molière soit — à sa façon, bien sûr - au moins l'égal de Shakespeare, cela ne me paraît aucunement contestable.

15. M. Paré. - Pour revenir au problème de fond demandons-nous : quand est-il acceptable de faire une adaptation au lieu de créer et qu'est-ce qu'il faut 
à cette adaptation pour qu'elle soit acceptable ? Je pense que c'est une question qui a deux aspects, d'abord l'annonce d'origine en soi peut-elle s'adapter ? Et une fois qu'elle est adaptée, on peut juger les qualités de l'adaptation. Cette solution de remplacement est, je ne dis pas un mal, mais une démarche nécessaire dans une foule de circonstances, de lieu, d'espace, de budget, d'argent.

16. L. Roy. - Je pense qu'on peut reconnaître que l'adaptation est parfois acceptable tout en admettant qu'elle soit un mal nécessaire dans notre contexte économique.

L'idée d'adapter plutôt que de créer vient d'une situation économique anormale... anormale dans le sens que le produit ou le service est souvent conçu ailleurs. Le produit est mis au point à distance. Avant qu'il soit mis sur le marché ici, la communication à son sujet est fortement engagée, une documentation a été amassée, un vocabulaire a été créé. Le produit a déjà toute une histoire dont on ne peut pas ne pas tenir compte. Si le produit était créé ici, ce serait bien différent.

Le publicitaire n'est pas totalement heureux d'envisager l'adaptation. Il se sentirait plus libre de toujours partir à neuf. Mais ayant à adapter, en somme, un produit au marché, avant de doubler les frais de production publicitaire, il doit se demander si la communication existante ne s'adapte pas... et il doit le faire objectivement, sentimentalité à part.

Toutefois, quand le publicitaire adapte, il ne doit pas rester prisonnier des faits contenus dans le message anglais. Il doit aller aux renseignements. Il faut créer à partir de l'adaptation. J'ai vu des adaptations publicitaires supérieures à l'original. N'ayant pas écrit le texte anglais, l'adaptateur d'expérience peut l'examiner avec objectivité et peut vraiment l'améliorer s'il sait s'imposer par sa compétence.

17. J.-F. Pelletier. - Ces renseignements dont il a besoin n'auront été fournis, en général, qu'au seul rédacteur d'origine, c'est-à-dire le rédacteur anglais. Quant au rédacteur français - au traducteur français, devrais-je dire - eh bien, mon Dieu! qu'il se débrouille, qu'il devine les renseignements nécessaires à travers le texte et l'image. C'est de l'idiotie carabinée, mais pourtant courante.

18. G. Normandin. - Cette idiotie, comme vous l'appelez, est de moins en moins courante, mais on rejoint ici ce que $M$. Roy disait tout à l'heure au sujet de l'origine étrangère du produit, ainsi que ce que vous disiez au sujet de la littérature, de la traduction littéraire qui nous fait entrevoir une facette de la culture étrangère à travers les œuvres de certains auteurs. Mais avec un produit comme «Coke », par exemple, quel mal y a-t-il à vendre le Coke à l'aide des idées des Américains, comme cela se fait partout en Europe, comme cela se fait dans le monde entier ou à peu près. Considérez aussi la campagne lancée par Esso, il y a quelques années : "Put a Tiger in your Tank».

L'idée du tigre m'apparaît comme étant une idée de vigueur, de force, de courage et cette idée est très facilement adaptable. D'ailleurs elle a été adaptée dans à peu près tous les pays du monde; je ne vois pas sur quoi on se baserait pour en condamner l'adaptation ici. 
19. J.-F. Pelletier. - Pour ma part, je n'accepte en rien le thème du «tigre », utilisé d'ailleurs, par plusieurs produits - et non pas seulement par Esso. Il y a quelques années, on m'y a mêlé pour une publicité de détersif. Après une étude qui me paraissait sérieuse et poussée, force me fut de recommander le rejet inconditionnel du «tigre» pour des raisons longuement expliquées. Mon client adopta et utilisa systématiquement, quand même, le «tigre » pour son savon. Aussi, pendant deux ans ou presque, ai-je systématiquement refusé de prendre au sérieux et d'approuver toute annonce basée sur le Tiger in Tide. En beau joueur, Procter \& Gamble me passa cette fantaisie qui, chez moi, prenait proportion d'une véritable crise de conscience publicitaire. Aujourd'hui, encore, je ne retire rien de mon refus du «tigre »; si c'était à refaire, je recommencerais sans hésitation aucune. Et je voudrais vous expliquer pourquoi. Surtout que ce thème du «tigre » illustre de façon dramatique les incidences du facteur socioculturel sur les publicités traduites ou adaptées. Au départ, disons que je ne nie pas au «tigre », évidemment, un certain succès de fait. Et ça ne changera pas d'un seul iota mon opposition au 《 tigre », pour la simple raison que le succès d'un thème, le succès d'une campagne, ne constitue pour moi qu'UN des critères appréciatifs. Critère d'une extrême importance, soit ! Critère qui finit souvent par être le seul pour bien des clients, hélas! Critère à placer en tête de liste, peut-être bien! Mais le succès ne reste que ça : un critère, un seul. Il y en a bien d'autres et, tout compte fait, de bien meilleurs.

Remontons au moment qui précéda le déclenchement de la toute première campagne Tiger. Ma condamnation d'alors reposait sur ceci. Le contenu sémantique de Tiger était tout autre que celui de «tigre». Le mot américain charriait alors un sens, un contenu métaphorique, que n'avait pas le mot français, et vice versa. Mais aujourd'hui, les publicités abusives d'Esso, de Procter et d'autres ont fini par faire croire aux gens que les recoupements sémantiques de «tigre » et de Tiger sont identiques - ce qui est complètement faux, au niveau des langues en cause. Quand la langue américaine utilise Tiger, elle veut souvent signifier dynamisme, rapidité, énergie, promptitude et primauté. Alors qu'en bon français, le «tigre » reste un animal féroce, sanguinaire, sournois et solitaire — bref, un être qui, à part sa force, n'a à peu près aucune qualité ; en tout cas, aucun trait qu'on puisse vraiment aimer et admirer. Bref, le Tiger anglais (pensez au sens social de he's a real tiger!) représente le chef énergique et rayonnant, quelqu'un qui a beaucoup plus de qualités que de défauts. Alors que le «tigre», en français, offre beaucoup plus de défauts que de qualités. Même chez Clemenceau... qui était le 《Tigre» surtout par son côté excessif, autoritaire - pour tout dire, hargneux et bête. Je respecte le tigre, je le crains volontiers; mais je n'en veux pas parce que je ne l'aime pas et l'admire peu.

Tout cela est tellement vrai que si on s'était arrêté un peu à une analyse comparative Tiger/tigre, une analyse fouillée et ouverte, on aurait bien fini par admettre que le contenu sémantique du Tiger anglais se retrouvait presque exactement - en français — non pas dans le tigre, mais dans le lion. Et à ce propos, je vous renvoie aux dictionnaires et aux bons écrivains - le Tartarin de Daudet, par exemple. Mais oui! le lion! l'être noble, fort, tranquille, serein, sûr de lui, 
puissant ; l'être à qui il ne manquait peut-être qu'un peu de vitesse pour équivaloir au Tiger américain.

Si je m'attarde tant à cette affaire, c'est qu'elle représente pour moi une supercherie majeure en adaptation publicitaire, ainsi qu'une pollution grave de cette partie de notre bien commun national qu'on appelle la langue. Nous en sommes au point où l'Anglo-Américain change le sens de nos mots français, les vide de leur contenu normal, et leur confère des recoupements métaphoriques qu'ils n'ont jamais eus. Cas typique, soit dit en passant, où le publicitaire québécois avait le devoir moral absolu de refuser net de participer à pareil gauchissement de la langue et de l'esprit français... (Mais combien de fois n'est-on pas un peu «p...», moitié par cécité, moitié par nécessité ?)

Et on croit me convaincre du contraire en me répétant que les campagnes Tiger/tigre ont de fait "réussi», que le consommateur québécois a fini par comprendre que «tigre» signifiait Tiger? La belle affaire! Ce n'est pas à moi qu'on apprendra la force bête, aveugle, inexorable d'un million de dollars consacré à un simple lancement de produit, dans la seule région de Montréal (un détersif l'a fait, il y a quelques années). Pareil budget peut faire passer la dernière des âneries - pour un temps, du moins. Mais ça ne change rien, rien ! à la nature de cette ânerie. Les âneries publicitaires à gros budget, mais il en pleut! Et plusieurs réussissent, semble-t-il. Et puis, après ? Car, c'est ça, le thème Tiger : une énorme ânerie à succès. Une ânerie (comme tant d'autres) qui trahit la société qu'on essaie de construire, qui fausse nos valeurs, qui change nos métaphores, qui abâtardit notre langue encore un peu plus... Et parce qu'elle a réussi, cette ânerie, on voudrait que j'applaudisse, que j'opine du bonnet? Le succès publicitaire, dans ces conditions, non seulement ne m'impressionne pas, mais je m'en fiche éperdument! Des traductions, des adaptations du type Tiger, ce ne sont pas des réussites, ce sont des crimes de lèse-langue et de lèse-nation. Et si on me demande de répondre de «façon réaliste» (!) à la question posée, je répondrai par une boutade qui est plus douloureuse que drôle : une adaptation est acceptable quand c'est un ordre absolu du client...

20. G. Normandin. - Je voudrais apporter une précision au sujet du tigre. Votre point de vue est intéressant, mais je ne le partage pas tout à fait. Vous restreignez votre interprétation du tigre au côté métaphorique américain, alors que pour moi, il acquiert une dimension différente en passant au Canada. Je vous concède le côté féroce du tigre, mais n'est-ce pas cela qui a accroché le côté féroce des automobilistes latins? L'automobiliste latin est un combattant; il prend le volant de sa voiture et il s'en va au combat. Et, en acceptant d'utiliser le symbole du tigre pour vendre de l'essence d'automobile, on a frappé dans le mille auprès de nos consommateurs latins. On dit que l'endroit au monde où la campagne a eu le succès le plus retentissant, c'est en Italie.

21. L. Roy. - Il me semble qu'il est un point fondamental où M. Pelletier et M. Normandin ne s'entendent pas du tout. Il s'agit de la question suivante : « Le seul critère à observer est-il la rentabilité de la publicité ? »

J'ai eu l'impression que votre appréciation était faite uniquement en fonction de la portée commerciale de la publicité dans les différents marchés où on l'utilise. 
Si c'était votre point de vue, je ne serais pas d'accord. Le publicitaire a, vis-à-vis du public, une responsabilité qui ne se limite pas à la vente du produit. Il doit tenir compte de la culture de ce public et ne doit pas pécher par mauvais goût. Le succès seul d'un produit ne signifie pas que la publicité est acceptable.

22. G. Normandin. - Si j'ai donné l'impression que c'était acceptable, je retire tout ce que j'ai dit sur le sujet. Ce que je crois avoir dit c'est que le premier rôle d'un publicitaire est de vendre le produit. Je rejoins M. Pelletier qui placerait ce critère en tête de liste. Quant aux autres critères, je pense les avoir inclus dans ma réponse à la question qui nous a été posée : si l'annonce se reconnaît comme une adaptation, un calque de l'anglais, elle est condamnable; si elle ne se reconnâit pas, si le consommateur s'identifie à ce message qu'on lui propose, c'est qu'il se produit en somme une certaine communication sur le plan de la culture.

23. J.-F. Pelletier. - Mais ne croyez-vous pas, M. Normandin, que votre théorie revient à dire que plus une adaptation donnera le change, c'est-à-dire donnera l'impression que c'est une création, plus cette adaptation est recevable?

24. G. Normandin. - Mais vous pouvez donner le change en faisant une annonce en joual; vous allez donner le change à votre consommateur. Mais votre consommateur va-t-il vouloir s'associer mentalement ou culturellement à ce message-là ? Il ne s'agit pas de jeter de la poudre aux yeux; il s'agit de traduire (et j'emploie le mot à bon escient), de traduire le message pour qu'il s'adapte à notre marché.

25. L. Roy. - Est-ce que ce n'est pas la trop forte quantité d'annonces mal traduites qui nous répugne? Les cas isolés d'annonces exotiques passeraient bien. Mais, il y a trop d'annonces où l'on ne se reconnaît pas dans le personnage, la situation, l'humour. C'est pour cela que le publicitaire québécois doit se reconnaître une responsabilité morale quand il vend un produit par la publicité.

26. J.-F. Pelletier. - Bien d'accord, M. Roy. Si on s'attend à ce que le consommateur achète le produit annoncé, c'est qu'on s'attend que ce produit soit pleinement accepté par lui; qu'il devienne même, en quelque sorte, partie de son être, de l'être qui va vivre avec ce produit ou ce service après l'achat. Si l'achat devient une chose aussi «personnelle», il est donc indispensable que le consommateur se reconnaisse dans les situations décrites dans la publicité. Autrement, pourquoi achèterait-il ?

27. G. Normandin. - En vertu du désir d'imitation...

28. J.-F. Pelletier. - Mais, alors, c'est que notre consommateur veut vivre dans sa vie comme les gens perçus dans les annonces vivent dans la leur. Mais, dans les deux cas, l'annonce en question doit lui permettre de se reconnaître - soit directement, soit par analogie. Autrement, c'est de la mauvaise publicité.

29. G. Normandin. - Pas nécessairement. Supposons que vous adressiez votre publicité à un niveau socio-économique moyen en présentant des situations de niveau socio-économique supérieur. Votre client n'a aucune chance de se reconnaître dans ces situations-là mais vous créez quand même chez lui le désir d'atteindre à cette situation, un jour... 
30. J.-F. Pelletier. - Un instant, on joue sur les mots! Du moment qu'on offre au consommateur la possibilité de se reconnaître "en puissance», dans une situation, cela suffit. Affaire de miroir, de face ou de biais, peu importe. Mais il faut qu'il lui soit possible de se reconnaître pour vrai, dans notre miroir publicitaire, que ce soit par identification absolue ou approximative. Ce qu'il faut rejeter, ce sont les annonces où aucune identification n'est possible. Par exemple, proposer des igloos comme habitation aux gens de Montréal. Pareilles présentations publicitaires sont à la fois bêtes et immorales : bêtes parce qu'elles ne conviennent pas au contexte, immorales parce qu'elles laissent entendre que l'identification consommateur/produit finira par se réaliser... par magie.

31. L. Roy. - Il faut utiliser la langue du consommateur dans la publicité. Autrement, on doit accepter le blâme collectif de porter atteinte à sa culture. $\mathrm{Je}$ crois que la communication n'appartient pas au fabricant, elle appartient à celui à qui elle est destinée. Qui plus est, je crois que la publicité est plus rentable ainsi...

32. G. Normandin. - Je rejoins M. Pelletier lorsqu'il accuse la publicité et l'adaptateur publicitaire de fausser souvent le climat culturel, de le transformer. Je le déplore presque autant que lui. Mais sans revenir à l'exemple du tigre, je crois que certains phénomènes se produisent en publicité qui permettent au consommateur canadien-français de transposer mentalement sur son propre plan l'exemple, la métaphore qu'on lui propose.

33. J.-F. Pelletier. - M. Normandin, sur la question du «tigre», un dernier commentaire. Je vous supplie d'accorder à la question suivante un maximum de lucidité et d'ouverture d'esprit. Croyez-vous vraiment qu'Esso et Procter \& Gamble auraient utilisé cette métaphore du tigre, si elle ne leur avait pas justement fourni les formulations exceptionnelles que l'on sait : «Put a Tiger in the Tank», pour Esso, et «Put a Tiger in Tide», pour P. \& G. ? Et qu'en fait, le point décisif fut la bienheureuse et mnémonique allitération de ces deux formulations ? Allitération fascinante en anglais... et inexistante dans les «adaptations » françaises des mêmes thèmes. Bref, la simple vérité exige qu'on admette que le « tigre », comme matériau publicitaire de base, n'offrait pas en français la plénitude naturelle qu'il fournissait en anglais. Somme toute, "Put a Tiger in the Tank» possède une clarté commerciale, une justesse linguistique et une puissance incantatoire que n'a jamais eu, et n'aura jamais, son pendant français « Mettez-y du tigre ». Du reste, à la limite, le thème français signifie très exactement rien du tout... surtout quand on l'applique au détersif !

34. G. Normandin. - Je suis d'accord avec vous pour ce qui est du savon, parce que l'élément de férocité dans le savon est un élément négatif...

35. M. Paré. - Roger Boivineau, peut-on faire de la bonne adaptation, et que faut-il pour en faire?

36. R. Boivineau. - Comme aurait dit M. de La Palice, pour faire une adaptation publicitaire, il faut d'abord avoir un texte et un traducteur. Il faut que ce traducteur ait des outils - sa documentation — et il lui faut du temps. Avoir un texte, cela 
veut dire un texte adaptable. C'est ce que nous avons essayé de définir jusqu'à présent. Partant de là, il faut avoir ensuite un traducteur ou un adaptateur avec toutes les qualités que cela suppose, c'est-à-dire la compétence du bon traducteur : bonne connaissance de la langue de départ; parfaite connaissance de la langue d'arrivée et de toutes ses ressources, avec l'aptitude à les exploiter, le tout en plus des qualités de publicitaire. C'est assez difficile à réunir. Ensuite, il faut les outils. Dans le cas de l'adaptation publicitaire, il faudrait avoir accès au moins aux mêmes renseignements que le rédacteur anglais, et cela n'est pas courant, même dans les agences, vous le savez. Ensuite, il faut avoir le temps et c'est un facteur extrêmement important. C'est peut-être en fait celui sur lequel on bute le plus souvent parce qu'on ne comprend pas que pour rédiger une annonce en français en partant de l'anglais il faut parfois presque autant de temps que pour la rédiger en langue originale. Enfin, il faut avoir la liberté d'agir, ce qu'on laisse de plus en plus aux traducteurs et adaptateurs mais pas suffisamment. En particulier, on leur impose trop souvent un parallélisme entre l'anglais et le français, ce qui constitue un obstacle insurmontable à la liberté de l'adaptation. Ce parallélisme ne se fait pas sentir souvent dans les annonces, parce que les annonces sont publiées séparément (sauf quand des éléments de l'annonce ont été présentés en parallèle par ailleurs; c'est le cas de certains thèmes publicitaires présentés dans la publicité sur le lieu de vente), mais dans les textes publicitaires bilingues; on veut absolument que le français colle à l'anglais. À ce moment-là, on fait de la traduction littérale mais pas de l'adaptation publicitaire.

37. J.-F. Pelletier - Bien d'accord avec tout ce qu'a dit M. Boivineau. Mes seules réserves sont toujours les mêmes : l'adaptation reste un mal nécessaire, pas plus ; n'en faire que sur l'ordre du client; la vraie solution, c'est la création publicitaire. Mais, dans tous les cas, il faut que l'annonce dise quelque chose et ne fasse pas comme Mackenzie King qui passa trente ans à vous dire en détail absolument rien.

38. M. Paré. - Est-ce qu'on n'a pas l'impression parfois que le rédacteur de langue anglaise n'en connaissait guère plus que celui qui est appelé à faire la traduction?

39. J.-F. Pelletier. - D'accord, mais comme l'annonce américaine tend à être aimablement décousue, cela ne vous autorise pas à en faire autant. Si le texte américain saute gentiment d'un sujet à l'autre, notre annonce française exigera plus d'ordre et de rigueur - quitte à modifier sensiblement les structures (ou l'absence de structures) du texte d'origine. Que l'Américain fasse l'annonce qu'il veut, selon les meilleures modalités de sa langue et de son esprit, et qu'il nous permette d'en faire autant selon nos modalités propres. Mais pour qu'il y ait adaptation possible, il faut qu'il existe des «ponts», au moins approximatifs, entre les réalités américaines exprimées à l'américaine, d'une part, et les réalités québécoises à exprimer à la française, d'autre part. Quand ces "ponts » minimaux n'existent pas, l'adaptation réussie est impossible. Exemple : la petite annonce du cirage Kiwi. Un gros policier à bajoues, le sifflet à la bouche, siffle. Le titre français, «Propre comme un sou neuf », laisse perplexe parce qu'il ne colle en rien à l'illustration. Mais on comprend aussitôt qu'on lit le titre original : «Clean 
as $a$ whistle »... Transposition parfaite du texte, et nulle quant à l'illustration. Il fallait rejeter le tout, ou changer l'image.

40. G. Normandin. - Je pense qu'on est tous d'accord ; on peut faire d'excellentes adaptations. Mais l'adaptation doit se faire dans les conditions que $M$. Boivineau vient d'énoncer. Il faut surtout que l'adaptateur soit un excellent concepteur publicitaire et qu'il ait le choix d'adapter l'annonce qu'on lui soumet ou de concevoir une annonce qu'il considère supérieure à celle-là pour son marché.

41. M. Paré. - Est-ce que vous considérez que l'imagination est un facteur important pour l'adaptateur?

42. R. Boivineau. - Absolument, et probablement le plus important. La bonne connaissance de l'anglais, la parfaite connaissance du français, cela va de soi, mais l'imagination créatrice est essentielle. D'après moi, à ce point de vue, il devrait y avoir peu de différence entre un bon concepteur publicitaire et un bon adaptateur publicitaire, sinon que le bon adaptateur publicitaire doit connaître l'anglais.

43. L. Roy. - Quand on dit que l'adaptation est possible, soulignons, par contre, que la plupart du temps le slogan, lui, s'adapte mal. Il a souvent une forme idiomatique et c'est peine perdue que de chercher à le traduire. Il est préférable d'ignorer totalement l'anglais et de créer un thème bien français.

MM. Paré et Pelletier ont rappelé que le texte anglais n'était pas toujours irréprochable. C'est bien mon avis. On a beaucoup trop tendance à excuser le texte anglais. Souvent l'adaptation ne pouvait pas marcher parce que l'idée d'origine n'était pas valable en anglais. De cela, on conviendra six mois après que l'annonce anglaise aura paru, mais pas sur le coup. Quand le texte anglais est insuffisant, je n'y vois pas là une question de culture ou de système de valeurs, c'est à mon avis, tout simplement aussi une insuffisance du concepteur anglais. Ce qui n'est pas rare du tout.

Pour en revenir à un point soulevé précédemment, j'accepte que l'adaptation soit un mal nécessaire parce qu'elle limite la liberté du rédacteur-concepteur. Cela rend son métier moins intéressant. Mais je crois tout de même, qu'en faisant de la création à partir de l'adaptation, on arrive à faire des choses intéressantes.

44. R. Boivineau. - Je pense à un mot qui résume peut-être ce qu'a dit M. Roy. L'adaptateur doit considérer l'annonce dans la langue de départ, simplement comme un canevas sur lequel il doit broder, il doit créer une nouvelle annonce.

45. M. Paré. - On peut maintenant passer peut-être aux obstacles et difficultés qu'on rencontre généralement dans les agences de publicités et dans les services auxiliaires des agences. Parlons de traduction si vous voulez et mentionnons également «adaptation» parce que pour moi une adaptation couvre une très vaste gamme, depuis la quasi-traduction littérale jusqu'à l'invention presque entière sans aller jusqu'à la création. Quels sont généralement les obstacles et difficultés que l'on rencontre?

46. R. Boivineau. - Ils sont nombreux et de nature très diverse. Je m'en tiendrai à quelques difficultés techniques. D'abord, celles qui se rapportent à l'espace et 
au temps dont on dispose pour communiquer le message. La longueur du texte, et celle des titres en particulier, pose de difficiles problèmes. L'anglais est une langue extrêmement ramassée. Le français exige en général plus de mots, et des mots plus longs pour exprimer la même idée qu'en anglais, même si on arrive par la pratique à faire d'excellents titres en français qui ne sont pas plus longs que les titres anglais. Mais à cause des contraintes typographiques, imposées par une maquette immuable, rédiger un titre revient parfois à faire des mots croisés. Il faut un certain nombre de lettres dans un certain nombre d'espaces. Voilà un obstacle important. Rappelons les difficultés déjà citées, par exemple l'illustration qui se rapporte à un jeu de mots, à une situation qu'on ne peut évoquer. Il faudrait avoir toujours la liberté de changer cette illustration. Il y a aussi les difficultés qui tiennent purement à l'information technique, par exemple lorsqu'on présente un produit nouveau. Le traducteur ou l'adaptateur ne sont pas toujours en possession des renseignements nécessaires et souvent aussi, le vocabulaire n'est même pas encore créé. Le traducteur doit donc créer rapidement des néologismes, des expressions nouvelles.

47. J.-F. Pelletier. - Pour ce qui est des difficultés techniques que rencontrent traduction et adaptation, je suis d'accord avec M. Boivineau. Et on pourrait sûrement en trouver d'autres. Mais je voudrais plutôt souligner deux difficultés qui, pour ne pas être techniques, sont peut-être plus graves et sûrement encore plus fondamentales. La traduction et l'adaptation publicitaires de qualité sont rendues quasi impossibles, au Canada français, par suite du profond manque de connaissances et de respect - de la part de l'annoncier - à l'endroit de la langue, de l'adaptateur et, à la limite, de la société à laquelle s'adresse le message traduit. Il ne s'agit pas, bien sûr, d'exiger qu'annonciers et agences connaissent couramment le français, adorent nos traducteurs, et soient des experts du milieu québécois. (Bien au contraire, puisque nous sommes là pour ça!) Mais il est grand temps que l'Anglo-Américain admette "pour vrai » qu'outre sa langue, il existe d'autres langues, aussi importantes et efficaces que sa langue ; qu'outre leurs spécialistes et techniciens, il en existent d'autres aussi compétents ; qu'outre leur société, d'autres sociétés belles et importantes existent... et qui méritent autre chose que de se faire drainer.

J'irais plus loin. Non seulement l'Anglo-Américain se fiche de notre langue, mais toute langue le laisse froid - y compris la sienne. Par le passé, j'ai volontiers cru l'Anglo-Américain "antifrançais »; à le connaître, je le considère "antilangues ». Et quand à la langue en tant que véhicule publicitaire, j'accuse spécifiquement l'annoncier et l'agent anglo-américains de croire dur comme fer que seul l'anglais est sérieux, seul l'anglais est «naturellement» publicitaire, seul l'anglais est efficace. Toutes les autres langues sont déficientes, de rendement inférieur; ce qu'elles peuvent faire de mieux, c'est d'être des calques de l'anglais. Bref, la vraie langue publicitaire, c'est l'américain ; aussi, la seule vraie publicité est-elle américaine. Et qu'en fait, Dieu a un pair, pour ne pas dire un père : il s'appelle Uncle Sam...

Les preuves de ces affirmations sont nombreuses. Rappelons seulement le fait de publier EN ANGLAIS des annonces dans des revues de France et d'Allemagne. Et, au pays, l'Anglo-Américain vous demandera sérieusement s'il ne serait 
pas plus judicieux, plus rentable, de supprimer vos annonces françaises pour n'y diffuser que des annonces anglaises. Mais, oui, voyons! Et pour deux raisons : il y a des stations bilingues, et tous les Canadiens français le sont aussi... La vérité, c'est que l'annoncier anglo-américain chérit les «piasses » du consommateur québécois, mais pas sa personne ni ses particularités socioculturelles.

48. G. Normandin. - Je vais vous surprendre, je vais abonder dans le sens de M. Pelletier... et également dans le sens de M. Boivineau. J'ajouterais une très grande difficulté à celles déjà énumérées : le manque de respect des AngloAméricains, comme on les a appelés, pour la qualité de nos concepteurs publicitaires. Et elle existe, cette qualité, même si nous devons déplorer chez nous le manque de compétences (au pluriel et non au singulier). Nous n'avons pas assez de professionnels de la conception publicitaire. Le jour où nous aurons ces professionnels, autre bataille à livrer pour prouver aux « autres » que nous sommes aussi bons qu'eux, on verra de moins en moins de ces horreurs publicitaires qui suscitent la critique et augmentent chez l'anglophone cette méfiance à l'endroit du français. Il faut au plus tôt organiser la formation de bons concepteurs publicitaires. Ces publicitaires professionnels feront disparaître plusieurs des difficultés que M. Boivineau a énoncées.

49. M. Paré. - Alors vous attribuez à la formation de bons publicitaires la possibilité qu'on leur accorde une mesure de confiance plus grande.

50. G. Normandin. - Oui, et également l'aplanissement des difficultés techniques que M. Boivineau a énoncées. Plus nous aurons de publicitaires compétents, plus ces difficultés-là seront atténuées.

51. L. Roy. - MM. Pelletier et Normandin ont fait allusion à l'attitude des Anglo-Saxons à l'égard de la publicité française. J'aimerais dire à ce sujet, que je n'ai pas connu dans mon milieu, d'attitude aussi fermée, aussi négative devant le désir du concepteur francophone de créer en s'éloignant de l'anglais quand il y a lieu. Je reconnais que beaucoup de rédacteurs francophones dans les agences prétendent ne pas avoir la liberté de créer. Je n'ai pas éprouvé cette difficulté. Je trouve que nos chefs de publicité de Toronto, autant du côté de l'agence que du côté du client, sont soucieux d'utiliser au Québec la publicité la plus convenable qui soit. Évidemment la vente du produit est ce qui les préoccupe le plus. Mais, en général, ils tiennent à savoir, dans chaque cas, si c'est par l'adaptation ou par la création originale qu'on peut le mieux atteindre le marché. Ils n'insistent pas pour qu'on produise une traduction si on leur dit qu'elle ne serait pas efficace.

52. M. Paré. - Ne croyez-vous pas que l'absence des Canadiens français compétents en publicité tient au fait qu'on leur fait remplir des fonctions auxiliaires au lieu de les laisser se consacrer à un domaine d'activités spécialisé par exemple dans les relations avec la clientèle, la recherche terminologique ou, si l'on veut parler d'un produit, la création et la rédaction. On en fait plutôt des réalisateurs de télévision, de radio, des producteurs, etc. Mais c'est parce qu'on charge les autres de la communication entre l'agence et le client, que l'on confie simplement au Canadien français les fonctions d'adaptation. 
C'est au concepteur français qu'il revient de recommander le genre de message à utiliser. Si la recommandation est bien faite, elle est généralement respectée. Seulement, pour créer, il faut du temps, beaucoup plus de temps que pour adapter. Il faut recueillir tous les renseignements, vérifier leur exactitude, établir une stratégie publicitaire et présenter soi-même le travail accompli. Souvent, le volume de travail constitue un obstacle à la création beaucoup plus grand que l'attitude du client torontois ou du chef de publicité unilingue. C'est un fait que, comparativement aux services anglais de conception, il n'y a pas assez de personnel en création française. Voilà la difficulté.

53. J.-F. Pelletier. - Je voudrais dire que j'envie la chance de M. Roy d'avoir échappé à ce tranquille mépris que l'annoncier américain me paraît porter à notre langue et à nos traducteurs. $\grave{A}$ ce propos, on rétorque toujours que ces choses-là se passaient «autrefois». Alors, aujourd'hui, « on a changé tout ça », comme dirait Molière! Au risque de scandaliser M. Roy, je souligne qu'une très importante maison torontoise faisait établir (il y a quelques semaines!) la déclaration annuelle du président par une jeune anglophone who knows French very well. Et je voudrais répéter que j'ai bien changé d'idée sur les motifs qui poussent l'Anglo-Américain à traiter ainsi notre langue de quantité négligeable. Autrefois, j'y voyais une mauvaise volonté lucide et agressive. Aujourd'hui, je ne crois plus à cette méchanceté; il s'agit plutôt, je pense, d'une ignorance aussi bête que profonde. Une ignorance qui les pousse à résoudre tout problème de traduction à la dernière minute et en s'adressant au premier venu, secrétaires et commis compris : «Do me a favour, will you? You know French, why don't you translate this for me? I'm in no rush, I'll wait...»

La vérité, c'est que l'annoncier anglo-américain n'a saisi en aucune façon les difficultés singulières et le cheminement délicat de ce processus magique et merveilleux (qui ne cesse de m'épater...) qu'on appelle traduction et adaptation. Au mieux, il les conçoit comme suit. Dans ce coin-là, il y a une grosse boite pleine de mots anglais; l'Américain en a la clef. Dans ce coin-ci, existe une autre boîte pleine celle-là de mots français ; le traducteur en a la clef. Au signal, chacun ouvre sa boîte respective, et l'Américain dit au traducteur : «I'll toss you a few English words, you toss back a few French words, and everybody'll be happy. » Non seulement c'est aussi simple que le baseball, c'est aussi niais. Ce qui n'est pas peu dire.

Le remède? Il faudra que la confrérie des traducteurs établisse un jour, pour les publicitaires anglo-américains, une traduction ad usum Delphini du petit livre de Paul Chauchard (publié dans la collection «Que sais-je ?») intitulé le Langage et la pensée. Ce texte deviendrait must reading pour tous ceux, là-bas, qui de près ou de loin s'occupent de publicité francophone. Parce que ce n'est qu'alors, peutêtre, qu'ils verront que : French is NOT only a language (which, in your evaluation, anyway, is nearly nothing). Like all great languages, French is also a way of thinking and, even, a way of life. Now, if you don't understand that, you understand NotHING! Bref, la description loufoque de pareil état d'esprit est bien donnée par ce G. I. américain revenu d'Europe et qui s'exclamait : «Do you know that Parisian kids are so smart that, even when they're only three years old, THEY ACTUALLY SPEAK FRENCH! 》Voilà qui décrit toute la situation. 
54. M. Paré. - Alors on en conclut que l'ignorance assez répandue dans le domaine de la publicité, peut-être même chez des gens qui occupent des postes d'une certaine importance, est un obstacle au sain épanouissement d'une publicité française... Maintenant je voudrais ajouter une question supplémentaire : Est-ce que souvent l'incompétence, disons de Canadiens français qui sont en poste chez le client, ne présente pas aussi un obstacle ?

55. L. Roy. - En général, on se plaint du manque de Canadiens français dans la haute hiérarchie du marketing, mais quand un francophone détient un poste d'autorité chez un client, le publicitaire se demande parfois s'il a la compétence voulue pour juger la publicité française. Personnellement, j'aime mieux le voir là que ne pas voir de francophone du tout. Je crois qu'il se fait plus de création originale française pour les clients de Montréal que pour les clients de Toronto. Quand le siège social d'une société est situé à Montréal, les exigences en matière de publicité francophone sont plus élevées, ce qui s'explique sans doute par le fait que les hommes du marketing français sont plus nombreux ici qu'à Toronto.

56. M. Paré. - Maintenant passons à la question suivante : On voit beaucoup de mauvaises adaptations, comment corriger cette situation ? Maintenant, il s'agit de trouver des solutions d'ordre général : initiatives ou recommandations pour améliorer la situation, proposer une intervention gouvernementale, que sais-je?

57. R. Boivineau. - Si vous le permettez, je voudrais simplement nuancer la question. On voit beaucoup de mauvaises adaptations, c'est sûr. Mais on pourrait dire «dans certains domaines», et les domaines visés sont ceux qui échappent en général aux agences de publicité. Il est certain que la publicité qui sort des agences ou qui est faite par des professionnels s'est beaucoup améliorée et il est rare qu'on voie des horreurs comme celles qu'a évoquées $M$. Pelletier tout à l'heure. Disons qu'on voit encore de la publicité médiocre, mais on voit aussi de la publicité médiocre en langue originale. Ce n'est pas une excuse, il est vrai, mais cela peut expliquer quand même certaines défaillances en français. La mauvaise publicité, les mauvaises adaptations proviennent d'une part de petits annonceurs qui font du travail d'amateur ou qui confient aux journaux et revues le soin de préparer leurs textes et, d'autre part, de grandes entreprises qui ont un service de publicité vraiment insuffisant du côté français. Je pense aux grands magasins qui publient la plupart du temps une publicité réellement mauvaise, parce qu'elle est préparée par des gens qui n'ont pas la formation voulue, qui ne sont pas suffisamment payés, ou qui n'ont pas le temps de faire leur travail. Les catalogues des grands magasins sont également très mauvais, ils fourmillent de fautes de toute nature. L'observation est valable pour la publicité directe qui se fait dans les mêmes conditions. Sans parler de la publicité sur le lieu de vente dont l'adaptation est souvent laissée aux bons soins des imprimeurs !

58. M. Paré. - Et à ce moment-là il ne s'agit pas uniquement de magasins de langue anglaise, des magasins francophones font la même chose, ils s'expriment de la même façon...

59. R. Boivineau. - C'est vrai, et cela s'explique. En principe, cette publicité devrait être rédigée directement en français, mais on part de documents anglais 
ou de documents mal traduits pour faire les annonces. En fait, l'anglais est omniprésent.

60. J.-F. Pelletier. - L'amélioration de la traduction, de l'adaptation et même de la création publicitaires procédera, à mon sens, d'une épuration et d'un raffinement de la profession. Aussi faudra-t-il instaurer, à tous les paliers un enseignement théorique et technique de la publicité - même si l'on sait d'avance que le secteur ne fournira peut-être pas 100000 emplois... Reste qu'il faut enseigner la publicité à ceux qui veulent en faire, et ne pas se contenter du système qui a prévalu jusqu'ici ; devenir forgeron à force de forger. La deuxième façon d'améliorer les disciplines publicitaires et parapublicitaires, indirecte celle-là, serait d'infiltrer le secteur de l'annoncier lui-même - y compris les services de commercialisation, de publicité, de relations extérieures, etc. Il faut y faire entrer des gens qui, à défaut de formation technique, auront l'humilité de reconnaître qu'ils ne sont pas capables, par exemple, de faire de la traduction - et, en second lieu, admettront qu'il faut confier la chose à un véritable traducteur.

En troisième lieu, il faudra aussi agir du côté du public. Il n'y a pas de raison que le simple consommateur ne devienne pas beaucoup plus critique du texte publicitaire, plus exigeant et plus revendicateur de qualité en ce domaine. C'est surtout à quoi servent des cours comme ceux que je donne à l'Université de Montréal. Et déjà, je crois, les fruits se font sentir. Une éducation généralisée du public québécois, pour l'éveiller aux choses de la langue en général et de la langue publicitaire en particulier, est difficile, soit ! mais possible et terriblement urgente! Il faut absolument que notre public arrête de se faire passer n'importe quoi, par n'importe qui, au détriment de son esprit, de son cour et de son gousset.

Et si on trouve que c'est trop demander, qu'on soit donc alors vraiment " réaliste». Qu'on supprime, non seulement le français, mais l'anglais aussi. Abandonnons carrément la communication langagière pour passer, le plus tôt possible, à une simple gestuelle accompagnée de grognements (beaucoup de Québécois y sont presque déjà...). Depuis longtemps, Toronto et New York nous disent : «As long as they understand, who cares?» $\mathrm{Si}$ vos gens comprennent windshield et bumper, pourquoi ne pas le dire? Vrai de vrai, tout cela est angoissant et minable. Si on ne prend pas dès demain les mesures draconiennes qui s'imposent, bientôt le Québécois ne parlera plus — ni le français, ni l'anglais. Il grognera. Il poussera son voisin, lui fera des signes. Et bientôt, on sera revenu aux petits dessins sur les murs de la caverne... Et les derniers Québécois, restés par miracle locuteurs francophones, auront fort bien compris, eux, que la publicité américaine - aidée de l'incommensurable naïveté et de l'incurie bredouillante de nos chers politiciens - achevait justement le génocide culturel du Canada français. On oublie trop qu'il y a plus atroce que de tuer les corps : c'est de pourrir les âmes. Madison Avenue et nos mini rois-nègres s'y connaissent. 